\title{
Venous Materials: Towards Interactive Fluidic Mechanisms
}

\author{
Hila Mor $^{1}$ Tianyu Yu ${ }^{2}$ Ken Nakagaki ${ }^{1}$ Benjamin Harvey Miller ${ }^{3}$ Yichen Jia $^{4}$ Hiroshi Ishii $^{1}$ \\ ${ }^{1}$ MIT Media Lab ${ }^{2}$ Tsinghua University ${ }^{3}$ MIT Mechanical Engineering ${ }^{4}$ MIT School of Architecture \\ ${ }_{1,3,4}$ Cambridge, MA $\quad{ }^{2}$ Beijing, China \\ ${ }^{1}\{$ hilamor, ken_n, ishii $\} @$ media.mit.edu 22yuty16@mails.tsinghua.edu.cn ${ }^{3,4}\{$ bmill, yichenj\}@mit.edu
}
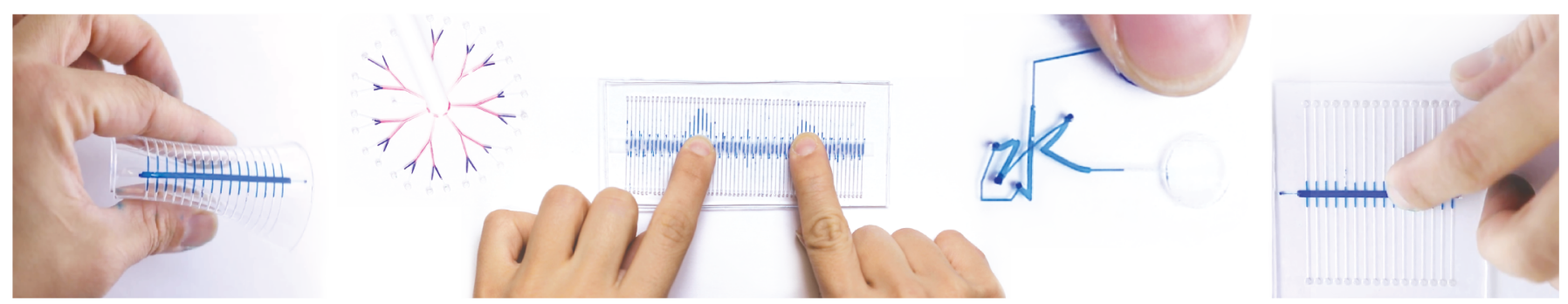

Figure 1. Venous Materials: a novel interactive material with fluidic structure that responds to tangible interaction.

\begin{abstract}
Venous Materials is a novel concept and approach of an interactive material utilizing fluidic channels. We present a design method for fluidic mechanisms that respond to deformation by mechanical inputs from the user, such as pressure and bending. We designed a set of primitive venous structures that act as embedded analog fluidic sensors, displaying flow and color change. In this paper, we consider the fluid as the medium to drive tangible information triggered by deformation, and at the same time, to function as a responsive display of that information. To provide users with a simple way to create and validate designs of fluidic structures, we built a software platform and design tool UI. This design tool allows users to quickly design the geometry, and simulate the flow with intended mechanical force dynamically. We present a range of applications that demonstrate how Venous Materials can be utilized to augment interactivity of everyday physical objects.
\end{abstract}

\section{Author Keywords}

Programmable Materials, Human Material Interactions, Microfluidics

\section{CCS Concepts}

-Human-centered computing $\rightarrow$ Interactive systems and tools;

\section{INTRODUCTION}

Venous structures are ubiquitous in nature. Beyond chemical and biological functionalities of the transmitted fluid, venous

Permission to make digital or hard copies of all or part of this work for personal or classroom use is granted without fee provided that copies are not made or distributed for profit or commercial advantage and that copies bear this notice and the full citation on the first page. Copyrights for components of this work owned by others than ACM must be honored. Abstracting with credit is permitted. To copy otherwise, or republish, to post on servers or to redistribute to lists, requires prior specific permission and/or a fee. Request permissions from permissions@acm.org.

CHI '20, April 25-30, 2020, Honolulu, HI, USA.

Copyright is held by the owner/author(s). Publication rights licensed to ACM

ACM ISBN 978-1-4503-6708-0/20/04 ...\$15.00.

http://dx.doi.org/10.1145/3313831.3376129 structures are a type of interaction that informs us of internal physical conditions in the body, such as the structure of the circulatory system. A venous system can also inform us of external conditions by changing its color. For example, the tip of our fingers blanch when we press firmly on rigid objects. We can find such color change as an interaction system throughout nature. Plants evolved with a system of veins and capillaries which can transmit water from roots underground to the leaves at the top of the tree. It can also transmit pigments that drive the color change of leaves [34], which then informs us not only of the condition of the leaf but also which season it is.

In past few decades, microfluidics have been a promising technology and research field [82] which aims to artificially create such fluid micro mechanisms. Lately, fluidic systems have been gaining even more interest across multiple disciplines for synthetic biological systems [3], for sensing methods [49, 55, $6,47,63,86,7]$, and for robotic actuation [33, 39, 14].

While microfluidic sensors, actuators and displays have been widely explored, we aim to embed programmable interactivity into venous fluidic mechanisms themselves. Our research intends to contribute to the HCI field by expanding the scope and design opportunity of interactive programmable materials research $[42,84,44,43,20]$ with the introduction of interactive fluidic mechanisms.

Specifically, our goal is to open a new material interaction design opportunity with a method of utilizing embedded fluidic mechanisms for display of mechanical forces and for augmenting direct tangible interaction. In this paper, we explore the design space of such fluidic mechanisms: we use the simple form of these mechanisms to visualize pressure. While such kinds of interactive design is commonly constructed with physical computing tools such as micro-controllers, sensors and actuators, our method integrates interactivity using embedded fluid mechanisms and channels. Therefore, our approach 
has the advantages of being based on simple self-contained systems, which do not require batteries nor rigid components.

This paper presents the design space and implementation of Venous Materials. We will present a set of geometric primitive patterns, and a flow vocabulary that constructs a toolbox for various tangible interactions with integrated fluidic mechanisms. We will show its functionalities and give examples of applications for integration in everyday objects and actions. In order to support HCI researchers and designers to prototype venous fluidic mechanisms, we implemented a software platform and UI tool for designing, modeling and simulating. We will also present our fabrication pipeline based-on laser cutters to engrave fluidic channels into polydimethylsiloxane (PDMS) sheets. Finally, we discuss the limitations and future work on how we envision Venous Materials to be expanded for the future of UI Design with rich input, output, and computation embedded in fluidic structures. Our contribution includes:

- The concept of Venous Materials that is a novel type of interactive material to embed interactivity in deformable materials with internal fluidic mechanisms.

- Design space which covers the basic architecture and the library of primitives for design of Venous Materials.

- Design and fabrication pipeline including specialized digital design/simulation tool and UI, and an evaluation of the simulation model.

- Demonstration of applications in everyday scenarios.

\section{RELATED WORK}

\section{$\mathrm{HCl}$ and Interactive Material Design}

HCI researchers have investigated how to shift interfaces away from screens and into physical objects and materials [23, 25, $80,69]$. While researchers commonly develop such interactive materials with computers combined with sensors and actuators [32, 35, 10, 24, 23], such systems rely on rigid and bulky hardware as well as real-time control software.

To tackle the complexity of these systems, HCI researchers have sought to develop non-rigid interactive material with means of computational fabrication, soft robotics, and material sciences $[54,5,4]$. Pneumatics have been widely explored for shape and stiffness changing interfaces [84, 44, 45]. In such research, the transformation pattern was embedded in the soft inflatable composite through simulation and fabrication methods. Generally, pneumatics still rely on rigid and bulky air pumps and micro-controllers, so researchers have explored embedding the interactive capabilities by encoding responsive behavior into material structures themselves. For example, by using hygromorphic or thermoplastic materials, researchers have proposed a method to design responsive shape changing behavior reacting to everyday interactions such as human-body sweat, heat of lamps, or even boiling water [85, 74, 73, 78, 11]. Another approach of material design with embedded interactivity is by designing the mechanical deformation properties that responds to external deformation and force. Metamaterial Mechanisms and KinetiX took this approach with architected hinge-mechanism to create intended deformation [20, 43]. Ion et al. extended their work to encode computational logic, and texture change to the fabricated material which expanded its interactivity $[21,22]$. HCI researchers have shown to program not only the mechanical deformation by design of internal structures, but also other interactive properties, such as programmable buoyancy, center of mass, and sound of wind instruments [77, 2, 52, 68].

The key idea for this branch of research is the introduction of fabrication methods and simulation models to create a responsive material that is encoded in the structure and property of material itself. This research space is defined and explored along multiple research concepts/paradigms, including Metamaterials [20, 48], 4D Printing [64] and Smart Materials [62, $70,83,75]$. As for such overlapped research realms, our work is intended to bring a novel approach of fabricating fluidic path within soft materials that is designed to be responsive to tangible and deformation input. This paper specifically seeks to explore mechanical deformation as input and color change as output as a first step towards this goal.

Color changing material have also been explored in HCI to enrich the interactivity of physical matter using triggers such as temperature $[31,56,72,40]$ and UV light $[58,29,16,53]$. In the materials science field, color changing materials responding to deformation has been explored as well [13, 59]. Our approach of using fluidic flow has a variety of design parameters for display patterns, mixing of colors and deformation inputs.

Recent HCI and Kinetic Art projects utilize fluids for interaction design and display $[41,67,15,50,37,60,65,71]$. Several projects explore computational control of the liquid flow in tubes and other fluidic paths to display information $[19,51$, 38], while we aim at controlling the fluid flow within a passive material reacting to deformation without any pumps.

\section{(Micro)Fluidic System and Design Toolkits}

In recent research in materials science, mechanical engineering, and computational fluidic systems, Microfluidics has been a topic that is widely explored [82]. For example, in Robotics, Microfluidics was applied to change the color of a soft robot for camouflage [39]. Microfludics with a chemically responsive material was used to develop robots with an entirely flexible body without any electric components [79]. SkinFlow utilizes transmission of liquids in a series of chambers to detect object deformation but with a camera [63]. Garrad et al. proposed a method to encode fluidic logic into a soft robotic system using conductive fluids inspired by biological veins [14]. One of their prototype integrated a transformation triggered by touch input with fluidic channel via electric actuation, which demands battery components.

There have been efforts to develop methods for researchers and designers to construct and customize their own microfluidics structures $[8,46,66]$. In our paper, we share our design and fabrication method utilizing custom developed simulation software as well as laser-cutter-based fabrication technique to design variety of responsive fluidic structures. Comparing to other simulation software of microfluidics [28], our software is intended to simulate the fluidic movement in reaction to 

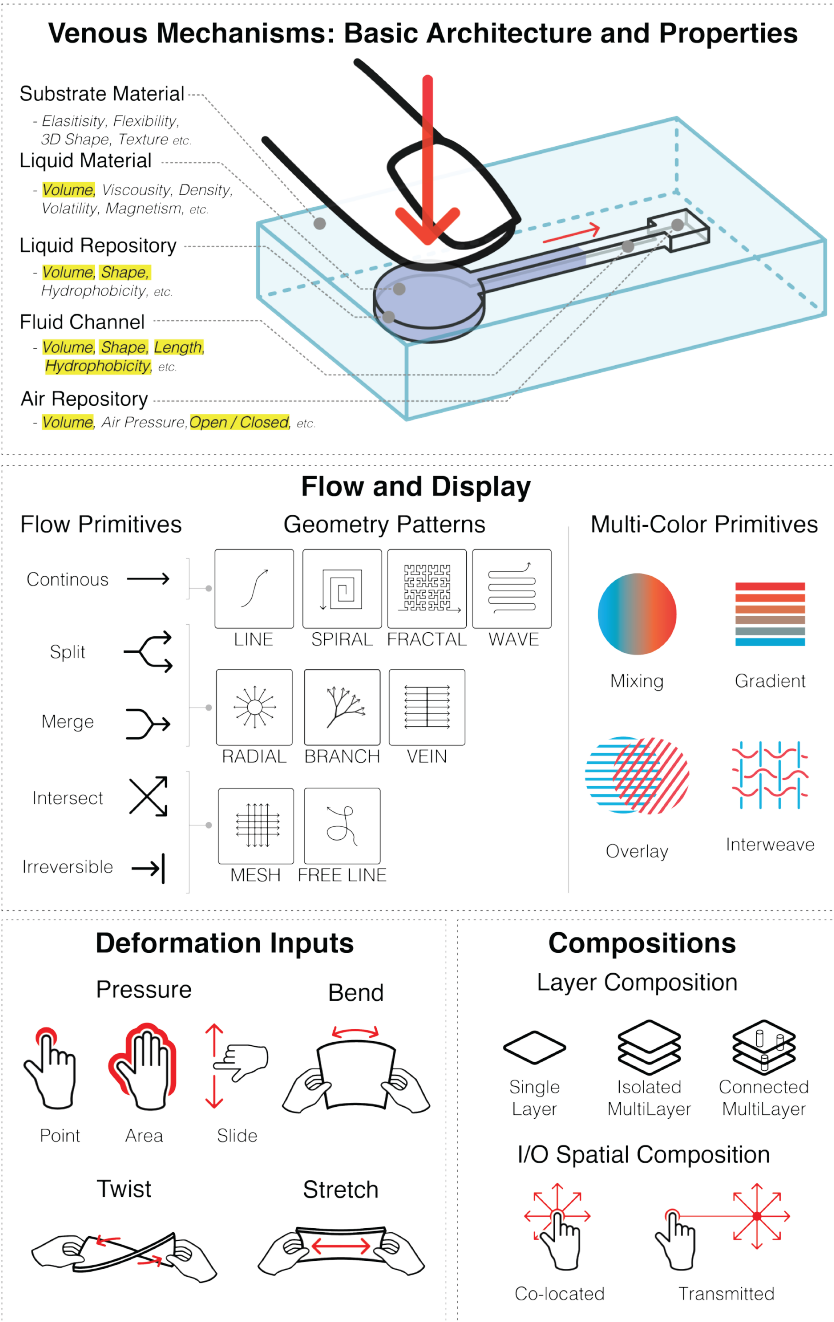

Functional Utilities
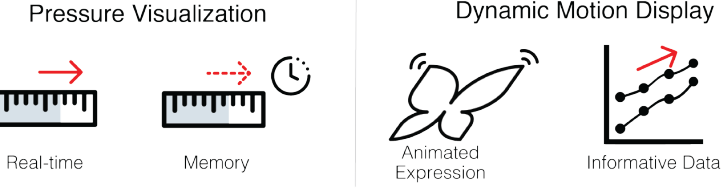

Figure 2. Design space of Venous Materials

deformable inputs for interaction design. While our design and fabrication was developed through a hands-on iterative process, we aim to not only convey the vision and concept of Venous Materials, but also to inspire designers and researchers to develop responsive fluidic materials with a relatively accessible method.

\section{DESIGN SPACE}

Venous Materials is a new approach to design interactive materials with means of designing fluidic channels inside deformable materials. The fluidic channels are designed to be able to respond to a deformation input in order to create dynamic visual feedback, enabled by flow of colored liquid. Cou- pling deformation input and flow output into a self contained integrated mechanism within a material is the key idea.

To explore this new category of interactive material design, we define a design space that overviews the design primitives of Venous Materials. Figure 2 presents the Design Space.

\section{Basic Architecture and Properties}

We first define the basic architecture of Venous Materials as shown in top of the Figure 2. Venous Materials is chiefly composed by two types of materials; Substrate Material, which is the external deformable substrate that has enclosed fluidic channels (here we use PDMS), and Liquid Material, which is the internally contained fluid that dynamically flows when deformation is applied (here we use water based ink). For the internal structure of Substrate Material where Liquid Material is contained, there are three major components. First, the Liquid Repository is designed to store the liquid in one area. In order for the fluid to flow out of the repository, the deformation and applied pressure should be focused mainly in the Liquid Repository. Second, the Fluid Channel is designed to connect to the Liquid Repository in an arbitrary path and geometry. The design of this path defines the display pattern of the material. Last, Air Repository is designed to be placed on the end of the fluid channel to control the air pressure of the internal structure. This enclosed Air Repository, is the key parameter that enables the fluid to flow back to the liquid repository after each deformation. The Air Repository can also be open to atmosphere, instead of enclosed in the $S u b$ strate Material which will make the flow irreversible after the deformation.

Size and dimensional ratio of the Liquid Repository, Fluid Channel, and Air Repository defines the sensitivity and scale of how the liquid flow in reaction to the magnitude of deformation. In our paper, for example, Liquid Repository is in milli-scale to contain a volume of Liquid Material, and Fluid Channel is in micro-scale to display dynamic movements of liquid flow. The way such a dimensional ratio affects the interactive properties motivated us to develop a design tool which can simulate the fluidic flow by taking into account the ratio of fluidic mechanism.

With this basic architecture, there are a variety of properties that can be customized and configured for each Venous Materials devices to create different types of interactive designs. For example, Substrate Material can take arbitrary 3D shapes to provide richer affordance design, or ferromagnetic liquid can be used as Liquid Material for a non-contact actuation. While, in this paper, we focus on the basic internal channel design that affects the fluid motion within a flat substrate material with colored liquid for visual output, we believe this basic architecture leads to much wider design space. The properties explored in this paper and the ones for future opportunity are represented in the basic architecture diagram in Figure 2, as the properties explored in this paper are highlighted with yellow.

Based on this core architecture, the rest of the design space presents the extended design primitives in four categories; Flow and Display, Deformation Inputs, Composition and Func- 


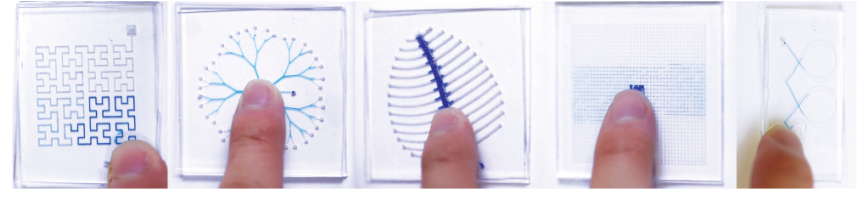

Figure 3. Sample Geometric Patterns (FRACTAL, BRANCHES, VEINS, MESH and FREE LINE).

tional Utilities. This design space is the core of this paper that bridges across following sections of the paper; Flow and Display as well as Deformation Input will be referred in our design pipeline, especially for simulation software design, and Functional Utilities will be demonstrated through multiple applications.

\section{Flow and Display}

One of the most important elements in Venous Materials is the capability of displaying dynamic information with visible flow. We define this display capability by introducing Flow Primitives, which demonstrates how a primitive channel can create different types of flow, Geometry Patterns, which describes how the Flow Primitives can be composed to create different geometric pattern, and Multi-Color Primitives, which utilizes multi-color in the fluidic channel.

\section{Flow Primitives}

Flow Primitives demonstrate forms in which a primitive fluid channel can create different types of flow. Continuous flow can be created with simple linear channel. Single channel can Split to multiple, while multiple channels can either Merge to one or Intersect.

\section{Geometry Patterns}

Based on the Flow Primitives, we designed multiple Geometry Patterns that offers different visual display outputs in the design, as pictured in Figure 3. As these patterns were designed to be utilized as a pallet (or swatches) by users to create complex fluidic geometry, the presented patterns are examples of many other possible ones. The different patterns are described in few major categories below.

The simplest category of Geometry Pattern is the continuous single line (e.g. LINE, SPIRAL, FRACTAL, and WAVE). This type of geometry simply maps the magnitude of deformation input to how far fluid travels in the single stream of flow. There is a category of patterns that utilizes the flow primitives, Split and Merge, to translate a deformation input to a dispersion display effect (e.g. RADIAL, BRANCH, and VEIN). These patterns can be utilized to create a display effect that can spread across a large area. Finally, there is a group of patterns that utilizes Intersect flow primitives. For example, MESH makes many intersections within a grid geometry, while FREE LINE makes intersections within a single continuous line. With this pattern, the Intersect primitive enables irreversible effect as shown in Figure 4a.

\section{Multi-Color Primitives}

Another important aspect for display capability is the color. Fluid with different colors can be used for Mixing to make color changes in response to deformation (Figure 4b). This

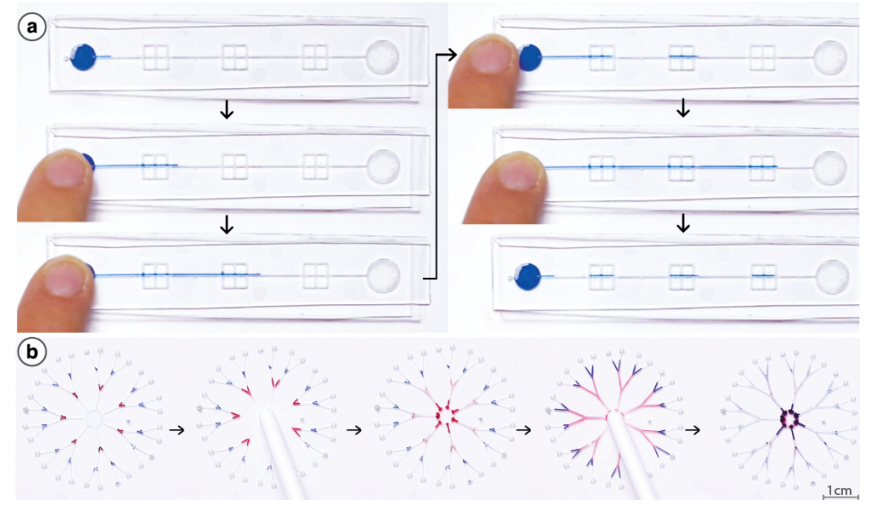

Figure 4. Two types of irreversible samples with a: MESH (Once the flow pass through the mesh, some of the fluid remains trapped in the channel, in three levels.), b: Color-Mixing (the mixed color is irreversible).

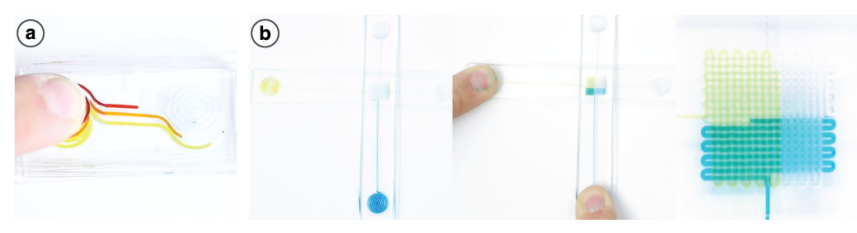

Figure 5. Multi-Color Primitives. a: Gradation, and b: Overlay

primitive is irreversible, which can be applied as a material with a mechanical memory. For reversible Multi-Color Primitives, fluidic channels with different colors can be composed in different ways including a Gradient by configuring the channels in order of gradient colors (Figure 5a), Overlayed to each other (Figure 5b) or Interweaved with multi-layer composition.

\section{Deformation Inputs}

Various ways of deformations can be applied as input to the fluidic mechanisms (Figure 6). This element is a key to trigger the fluid to flow within the channel. We define four major types of Deformation Inputs in Venous Materials; Pressure, Bend, Stretch (i.e. tension) and Twist (i.e. torsion). For each input, the magnitude of deformation affects the physical motion of liquid.

Pressure input simply represents an input made by pressing the material. Pressure can be applied in different types of spatial modalities, including Point (i.e. pressing with a finger tip), or Area (i.e. applying force with a palm or foot). The pressure effect can also be shifted across a continuous surface which can be described as Slide. For the Pressure input, the

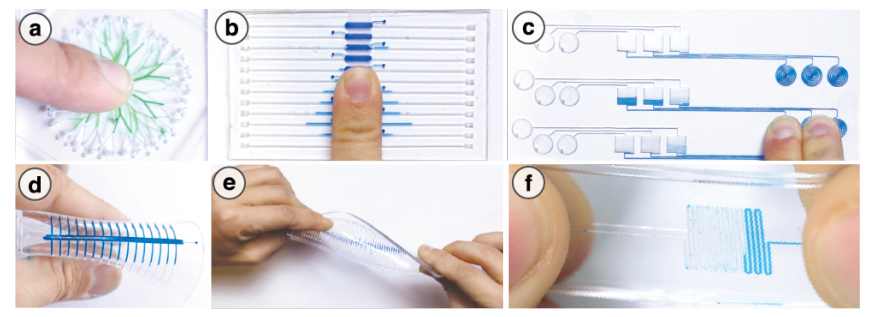

Figure 6. Deformation Inputs, Pressure for a: Point, b: Area, c: Slider, and d: Bending, e: Twisting, f: Stretching. 
magnitudes as well as location of the pressure affects the fluid motion.

Input actions that are afforded by Venous Materials' deformable sheet form factor can also be the trigger for fluid flow. Bend is one such example. The bending curvature and angle can be mapped to the magnitude of flow within the channel. Twisting and Stretch can also trigger the flow when applied as deformable inputs.

\section{Compositions}

As Venous Materials are composed of layers of flat sheets, different types of Composition can extend its simple structure and expand display and interaction capabilities.

The first type of composition is the use of multiple layers. While Single Layer Venous Materials is relatively easy to fabricate, it has certain limitations (e.g. the liquid cannot be overlayed without mixing). With Isolated Multi-Layer composition, Venous Materials can have Overlayed multi-colors, or with Connected Multi-Layer, the liquid can move across multiple layers to create complex pattern like the Interweaved multi-colors.

The second type of composition is the I/O Spatial Composition. The location of deformation input relative to that of the fluid display can be composed in either Co-located on the same location, or Transmitted apart from input. The composition of Co-located can be useful for users to intuitively understand the relationship of I/O, while Transmitted can be useful for users to see the display that is not occluded by the deformation source (e.g. pressure under a shoe).

\section{Functional Utilities}

We define how Venous Materials can be utilized for interaction design as Functional Utilities. This section describes how the responsive flow and display capabilities of Venous Materials can be interpreted and used for functional purposes in HCI and interaction design.

\section{Pressure Visualization}

In HCI, informing force and pressure between objects and objects / objects and bodies using electrical components is commonly explored for the area of tangible and embodied interaction [9]. Such a system is intended to help users understand the invisible data of weight, force or weight balance to improve their understanding to physical phenomena. Venous Materials can offer such Pressure Visualization utility only with self-contained material structures without any computational and electric components. The magnitude of deformation input can be mapped to fluid flow in Real-time which dynamically changes according to the amount of input. It can also be designed to visualize a force over time which can act as Memory. By utilizing some of the irreversible design primitives (e.g. Intersect or Color mixing), the stored fluid can be interpreted as a memory that the material stores when a certain amount of force is applied.

\section{Dynamic Motion Display}

Another category for the utility is for displaying different types of dynamic information with the motion of fluid. Specifically, two types of dynamic information can be displayed;
Animated Expression, which is to create animation with semantic expression (e.g. flapping butterfly with color change), and Informative Data, which is to inform abstract data with dynamic motion (e.g. line chart that expresses variation over time). These utilities will be demonstrated in-depth in the application section.

\section{DESIGN AND FABRICATION PROCESS}

In this section, we overview the pipeline of the design, simulation, and fabrication for Venous Materials (Figure 7). The pipeline starts from Design and Simulation of the venous structures, followed by a fabrication process based-on laser engraving PDMS sheets, manual injection and sealing.

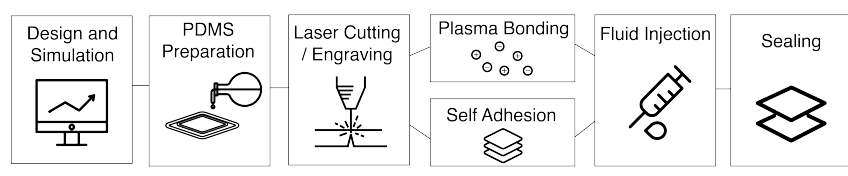

Figure 7. Design and Fabrication Pipeline of Venous Materials

\section{Design Tool and UI}

We implemented a specialized design tool which enables users to model, simulate, and adjust the venous structures. Users can simulate the flow response and fluid distribution under different deformation inputs and export the files for the laser cutting / engraving process.

The way Venous Materials respond to a deformation input is affected by different parameters of the materials and geometries. The main motivation of the GUI design tool is to provide users with a method for real-time simulation to support the iterative design of functional fluidic mechanisms.

Researchers in HCI have previously shown the benefits of design of specialized computational editors for simulation and design of programmable interactive materials $[21,20,85,44$, $42,74]$. Our design tool builds upon this HCI stream, however, it is specifically designed for fluidic structures and flow. While computational simulations are increasing in popularity in the microfluidics field [76, 28], our design tool specifically focuses on the simulation of fluidic flow in reaction to deformable input for interaction design purposes.

\section{User Workflow}

Our design tool is implemented in Rhinoceros 6 and Grasshopper environment. In this section, we give an instruction through an example user workflow. Figure 9 illustrates the step-by-step design and simulation workflow for our example prototype; a double layered mechanism that displays two different colors in response to bending. Each step in Figure 9 shows only the relevant section of our GUI, instead of showing the full GUI window.

(a) Plan and Design: A user starts the design by choosing a linear primitive geometry pattern that the user would like to generate from the swatch of geometry patterns. Once the desired geometry pattern is chosen, the user can configure the geometry in two simple steps. First, the user draws and defines the area in which the geometry will be generated. This step allows them to easily design and generate patterns across large 


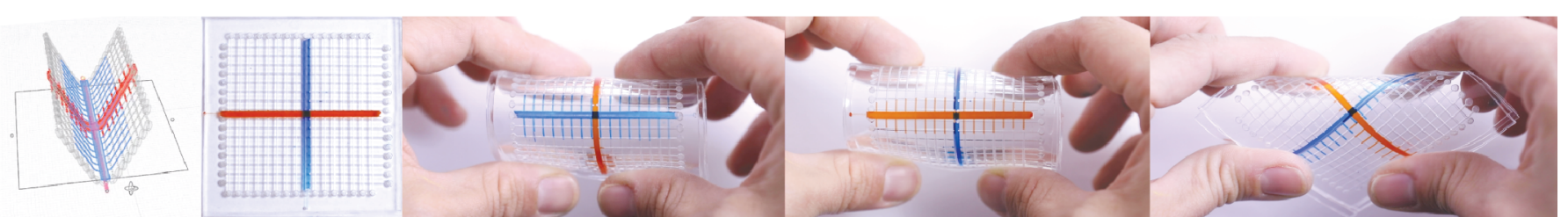

Figure 8. Simulation view and actual prototype of double layer linear prototype of Venous Materials. Fabricated prototype shows functionality of displaying two colors depending on the deformation.

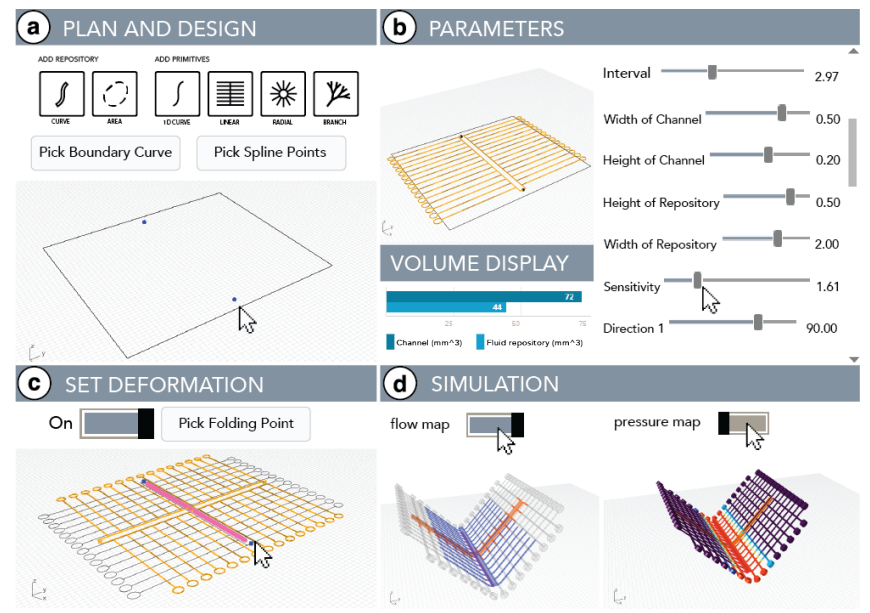

Figure 9. Design and simulation workflow of the prototype on Figure 8.

areas. Second, the user defines the flow direction by setting a starting-point of the flow (and in some cases an end-point as well). Once the two steps are done, the chosen primitive is automatically generated and displayed on the design view. The design tool is also capable of generating different types of geometries in a single model by repeating the steps above. Additionally, the software offers a free line drawing function where users can compose arbitrary linear shape of fluidic channel with a cursor. This function also lets users connect multiple geometric patterns.

(b) Adjust Parameters: Next, the user can adjust a set of parameters to define the detail parameters of the geometry of fluidic path. There are two major categories of parameters the user can adjust. One is the parameter of the primitives in the basic architecture including Liquid Repository, Fluid Channel and Air Repository. Properties including dimensions (e.g. size, width, and depth) for each primitives can be adjusted. The other category of parameters is for each geometry pattern, which are variables defining the global geometric configuration rather than primitive property of channels. The types of parameter depends on each geometry pattern, but they include density of patterns, orientation of the channels / patterns, etc. In this example, the design and parameter adjustment steps are repeated to generate a second layer.

(c) Set Deformation: When the design of the fluidic structure is complete, we set a virtual deformation input to the model of the fluidic channels. For folding deformation, as shown in this workflow, the user defines a folding line, folding curvature and folding angle. For pressure, the user defines a pressing point, a radial range of pressure, and a force.

(d) Simulate and Display: To simulate, the user turns on a simulation mode to display a Pressure Map and a Flow Map as a result. The Pressure Map view allows users to see a color-gradient map of the gradient of pressure intensity that results from the deformation input. In the Flow Map, users can view the simulation result of fluid distribution through the channels according to the deformation input parameters. This also allows users to view the change of overlapped colors in multi-layer composition. During the simulation mode, users can still modify any parameters from (a)-(c) steps. Users can view real time updates of the simulation and make iterations on their design. Finally, the design tool generates multiple DXF files depending on the fluidic channels and repositories with different depth. This is useful for the laser engraving step where each depth should be processed separately.

\section{Other Design Tool Capabilities and Considerations}

Multi-Layers: Our design tool supports multi-layer structure as well. The user can design and adjust primitives in different layers in the design step and also manually define connection between multiple layers. Simulation and actuation can also be applied for multiple layers together.

Fluid Volume Verification: Our design tool supports users to verify the volume of the fluid repository that can contain a sufficient amount of fluid for intended flow despersion. The user may take this parameter in consideration during the step (b), when simulation result of Flow Map view lacks the distribution of liquid flow.

Sensitivity Control: An additional parameter in the design tool is the control of sensitivity, which we define as the flowresponse-ratio according to deformation input. The sensitivity is defined by the ratio between the volume of the channels to the volume of the air repositories. To realize this function, when the sensitivity value is tuned by the user, the system adjusts the volume of the air repositories accordingly.

\section{Fabrication Method}

In this section we explain the fabrication process of the Venous Material structures. The key points for our choice of fabrication method and materials focused on a quick, low-cost, accessible, and scalable process. The material we used as the substrate is PDMS (Sylgard 184), which is widely used in the microfluidics field and allows us to follow existing published protocols on mixing, casting and molding [1]. 
A basic structure of Venous Materials is composed with three layers of PDMS; a bottom layer with the fluidic channels and repositories, a middle layer that has a hole for fluid injection, and a top layer that is a plain sheet for sealing. For multi-layer composition, multiple sets of bottom and middle layers were stacked with single top layer on the top. In our prototypes, we used 0.9 or $1.5 \mathrm{~mm}$ thick PDMS for bottom layer, and 0.5 or $0.9 \mathrm{~mm}$ thickness one for middle and top layer.

The fabrication process starts with creating the PDMS sheets by mixing, degassing and casting into prepared molds. We then cure the PDMS by heating to $27^{\circ} \mathrm{C}$ for 40 minutes, or leaving at room temperature for 24 hours.

We use a CO2 laser cutter and engraver (GCC Laserpro Spirit) for fabricating internal structure. This is the process to engrave fluid channel and repositories on the bottom layer. Based on previous characterization of laser cut PDMS [18, 36], we created our own library of channel and repository samples by changing engraving parameters, that affect the surface texture and fluid flow. Figure 10 shows a set of parameters that we optimized for our process. Other fixed parameters are: manual color fill mode, 1000 DPI, 200 PPI, and raster activated.

\begin{tabular}{lllll}
\multicolumn{4}{l}{ Laser engraving parameters library } \\
\hline Engraving & Depth & Speed & Power & Repeat \\
Repository & $0.9 \mathrm{~mm}$ & 45 & 75 & 2 \\
Repository & $0.45 \mathrm{~mm}$ & 45 & 75 & 1 \\
Channel & $0.1 \mathrm{~mm}$ & 60 & 60 & 1
\end{tabular}

Figure 10. Laser engraving parameters library.

After laser engraving, the engraved PDMS has to be cleaned with isopropyl alcohol (IPA) to remove grease, and tape to remove dust. We manually punch a hole to the middle PDMS layer based on the position of the fluid repository accordingly. Then, we manually align and layer the bottom and middle layer. We used two different methods for layering and bonding the layers in this process. The first is simply utilizing its self-adhesive properties, useful for rapid prototyping and reinjection of the fluids. The second method is using plasma bonding for higher adhesion between the layers. This is more efficient for condensed patterns and larger deformation input forces. After the layering we manually inject the fluid (Ecoline water-based inks) through the holes on the middle layer. Lastly, the whole composition is completed with seal using top layer of PDMS.

\section{SIMULATION IMPLEMENTATION}

Our simulation method aims to calculate a dynamic flow map for the venous structures according to the deformation input, using both theoretical analysis and experimental results. The core part of this method is a comparison of two pressure maps, which we will refer to as the Resistant Pressure Map and Actuated Pressure Map.

\section{Simulation Workflow}

This section gives a brief description of the workflow of our simulation method that was part of our design and simulation tool (See Figure 11).

Division into Cells: We first divide the channels into cells and use a data tree structure to store them. For each cell, we

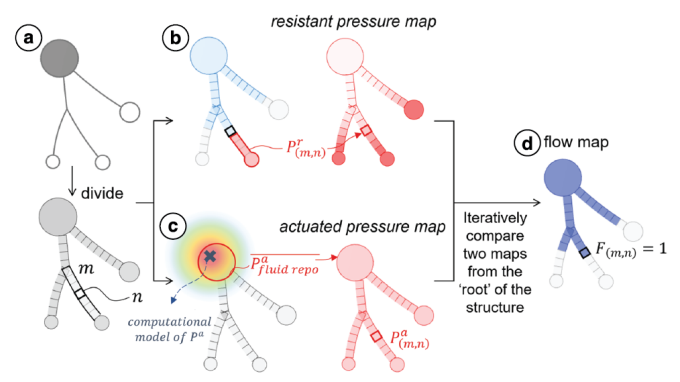

Figure 11. Simulation workflow. (a) Division into cells. (b) Calculation of resistant pressure map. (c) Calculation of actuated pressure map.(d) Calculation of flow map.

use a coordinate $(m, n)$ to describe its location, where $m$ is the index for the branch where the cell is located in the tree structure, and $n$ is the sequence index for the cell inside the branch (Figure 11a).

Calculation of Resistant Pressure Map: Next, we define a Resistant Pressure Map $P_{(m, n)}^{r}$ to evaluate the resistance of the venous structure to fluid movement. This resistance comes from an increase in the internal pressure when the fluid flows further into the channel, and compressing the air. We use Boyle's Law to calculate the internal pressure $P_{(m, n)}^{r}$ for each cell $(m, n)$ at the moment when the fluid reach that cell, which represents the magnitude of the internal pressure that is required in order for the fluid to travel to the cell (Figure 11b).

Calculation of Actuated Pressure Map: Then, we define an Actuated Pressure Map $P_{(m, n)}^{a}$ to evaluate the increment of internal pressure caused by the external actuation. The key point in this step is to calculate the incremental internal pressure of the fluid repositories $P_{\text {fluid repo }}^{a}$. We make this assertion for two reasons: 1) the fluid repositories are the source of a flow map, so in most cases the actuation is applied close to the fluid repositories; 2) we assume that we could equalize the fluid pressure in the channels to the fluid pressure in fluid repositories, as long as they are connected together, so that we can get the whole actuated pressure map $P_{(m, n)}^{a}$ after calculating $P_{\text {fluid repo }}^{a}$.

To get $P_{\text {fluid repo }}^{a}$, we use computational models to characterize the relation of the incremental internal pressure $P^{a}$ to the actuation parameters, which we selected as pressing pressure $P_{\text {press }}$ and folding curvature $R_{\text {fold }}^{-1}$. In the computational models, we first infer the proportional relation of $P^{a}$ to $P_{\text {press }}$ and $R_{\text {fold }}^{-1}$ with fundamental physics analysis, and next introduce a Gauss factor to describe the decay of the effect of actuation caused by the plasticity of PDMS. The integrated computational models are shown as Equation (1)(2), where $k_{p}$ and $k_{f}$ are two proportional coefficients, $d$ is the distance off the central actuation position and $\sigma$ is a decay coefficient.

$$
\begin{aligned}
P^{a} & =k_{p} P_{\text {press }} e^{-\frac{d^{2}}{2 \sigma^{2}}} \\
P^{a} & =k_{f} \frac{1}{R_{\text {fold }}^{2}} e^{-\frac{d^{2}}{2 \sigma^{2}}}
\end{aligned}
$$



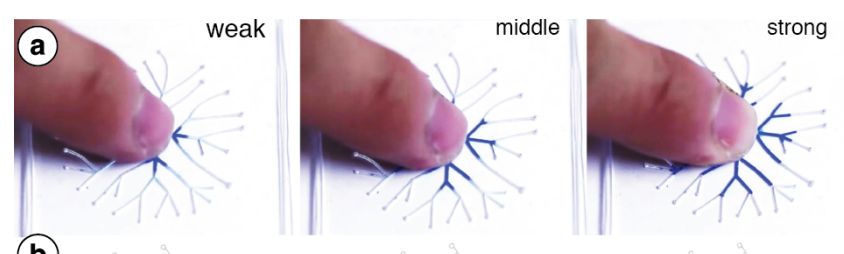

(b)

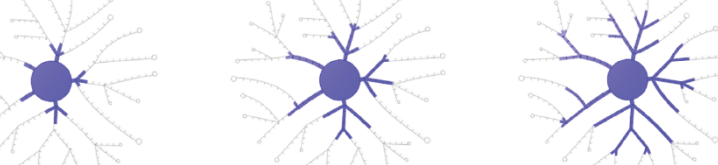

Figure 12. Experiments to test the resistance property of the venous structure on the fluid movement: (a) Experiment results. (b) Simulation results
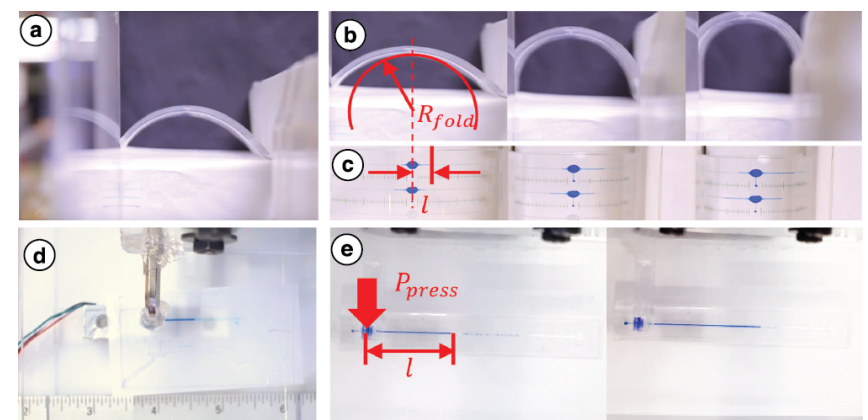

Figure 13. Experiments setups. Top:experiment of folding actuation. (a) Experiment set up. (b) Side view of experiment process. (c) Top view of experiment process, and data collection of folding curvature $R_{\text {fold }}^{-1}$ and fluid travel distance $l$. Bottom: experiment of pressing actuation. (d) Experiment set up. (e) Experiment process, and data collection of pressure $P_{\text {press }}$ and fluid travel distance $l$.

The undetermined coefficients above are later estimated with a few experiments, and then these models are used to calculate $P_{\text {fluid repo }}^{a}$. (Technically, for each fluid repository, we calculate the average of incremental internal pressure $\overline{P_{\text {fluid repo }}^{a}}$, shown in Figure 11c) These computational models are also used to display the pressure map in the simulation mode (Figure 9d,f).

Calculation of Flow Map: Once we have both the resistant pressure map $P_{(m, n)}^{r}$ and actuated pressure map $P_{(m, n)}^{a}$, we use an iteration algorithm to calculate the flow map $F_{(m, n)}$ (Figure 11d). For each cell $(m, n)$, the solving process is shown as Equation (3) where $F_{(m, n)}$ is a Boolean value representing whether the cell $(m, n)$ is filled by fluid, precursor of $(m, n)$ is the adjacent precursory cell of $(m, n)$ in the data tree.

$$
\begin{array}{r}
F_{(m, n)}=1 \text { only when } \\
\left\{\begin{array}{l}
P_{(m, n)}^{a}+P_{0} \geq P_{(m, n)}^{r} \\
F_{\text {precursor of }(m, n)}=1
\end{array}\right.
\end{array}
$$

\section{Experimental Modification and Evaluation}

We designed a few experiments to test the models we used in simulation, and to estimate the undetermined parameters. The experiments were conducted in the following stages:

- Experiments on resistant pressure map: test the resistance property of the venous structure on the fluid movement.
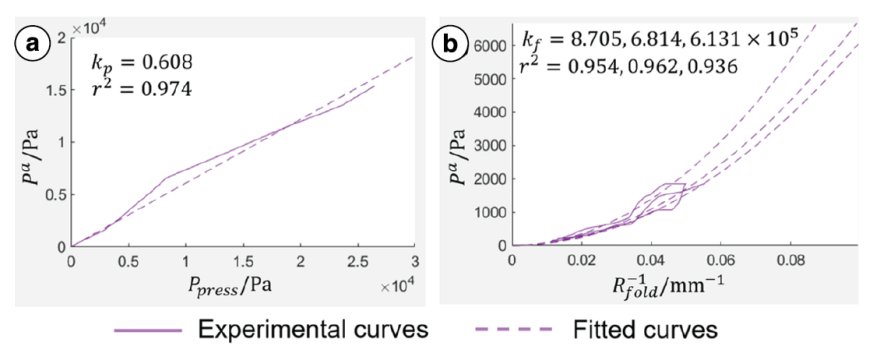

\begin{tabular}{|c|c|c|c|}
\hline \multicolumn{4}{|c|}{ Coefficient Estimate Results } \\
\hline \multirow{3}{*}{$\begin{array}{c}\text { Proportional } \\
\text { coefficients } \\
k\end{array}$} & Experiment & Average & Standard Deviation \\
\hline & Pressing $k_{p}$ & 0.614 & 0.291 \\
\hline & Folding $k_{f}$ & $6.513 \times 10^{5}$ & $2.329 \times 10^{5}$ \\
\hline \multirow{3}{*}{$\begin{array}{c}\text { Decay } \\
\text { coefficients } \\
\sigma\end{array}$} & Experiment & \multicolumn{2}{|c|}{ Estimated equation } \\
\hline & Pressing & \multicolumn{2}{|c|}{$\sigma=\frac{1}{2} \times$ pressing diameter } \\
\hline & Folding & \multicolumn{2}{|c|}{$\sigma=\frac{1}{2} \times R_{\text {fold }} \times$ folding angle } \\
\hline
\end{tabular}

(c)

Figure 14. (a) Fitting results of $P^{a}-P_{\text {press }}$ curve from one device in pressing actuation experiment. (b) Fitting results of $P^{a}-R_{\text {fold }}^{-1}$ curve from one device in folding actuation experiment. (c) Estimate results of the undetermined coefficients in the computational models (Equation 1,2)

- Experiments on actuated pressure map: test the computational models (Equation 1,2) and estimate the undetermined parameters.

\section{Experiments on Resistant Pressure Map}

We conducted an experiment to test the resistance property of the venous structure on the fluid movement. We used an asymmetric branch structure as our experimental device and applied a pressure gradually to the central fluid repository. We conducted this process both in our design tool and in an actual experimental environment. We then compared the simulation result with the experimental result. Figure 12 shows the comparison, where we find our simulated changing process is in close agreement with the actual changing process. This result shows a high coincidence of the resistance property between the simulated and actual performance, which proves the validity our resistant pressure map model.

\section{Experiments on Actuated Pressure Map}

We next conducted a few sets of quantitative experiments to test the computational models (Equation 1,2) and estimate the undetermined coefficients in them.

To experiment pressing actuation, we placed the sample (we used a single linear channel) on a load cell (TAL220), and applied force on the fluid repository gradually $(0.1 \mathrm{~mm} / \mathrm{s})$ with a linear actuator. Through recorded video, we noted data of the fluid travel distance $l$ and the pressure $P_{\text {press }}$ (figure 13d,e). We conducted this process on few devices with different dimensions, and repeated on each device three times. We picked 5 devices for data analysis. The data analysis process for each device follows three steps: First, we Boyle's Law and Equation (3) to reverse the travel distance $l$ to the incremental internal pressure $P^{a}$. Second, we use linear interpolation to get three continuous $P^{a}-P_{\text {press }}$ curves for three tests of each device, and calculate the average of three curves to minimize 

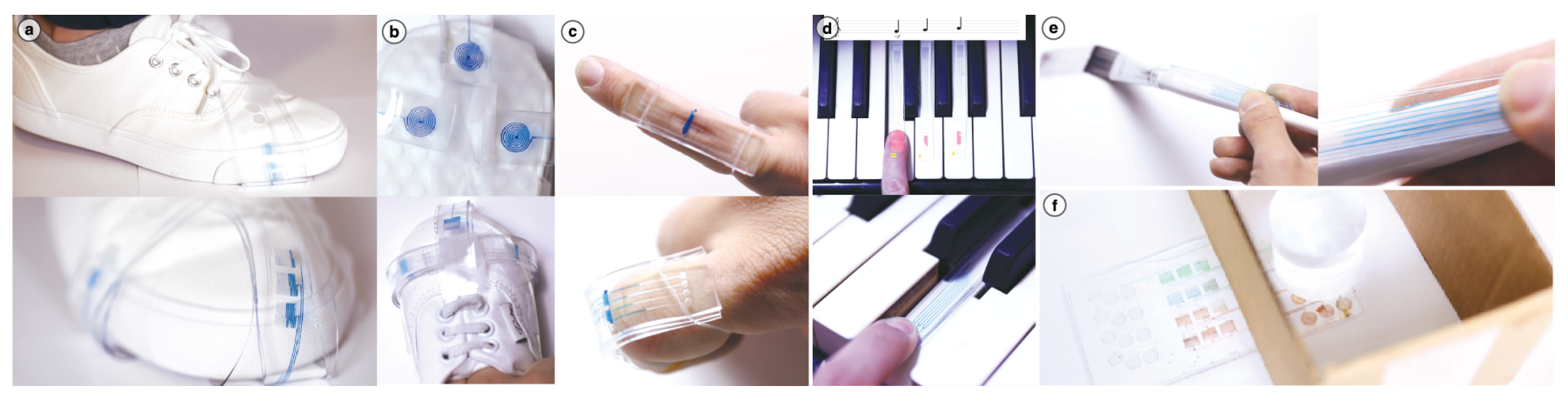

Figure 15. Applications of Venous Materials includes visualisation of (a, b) pressure distribution for shoe-soles, (c) bending angle of finger joints, (d, e) pressure applied to piano keys and brushes, (f) internal object condition of a package.

errors. Third, we fit the averaged $P^{a}-P_{\text {press }}$ curve with a linear proportional model to estimate $k_{p}$ in Equation (1).

For the folding experiment (figure 13a,b,c), we set up the device on a stage between two side-boards, one is fixed inplace and a second mounted to a linear actuator. By gradually moving the linear actuator, we fold and unfold each device, repeating three times. By recording video using two cameras in two different angles (top and front), we collect data of the fluid travel distance $l$ and maximum folding curvature $R_{\text {fold }}^{-1}$. Then, we calculate the average of linearly interpolated curves for both folding and unfolding for each test, and used a quadratic proportional model to fit the $P^{a}-R_{\text {fold }}^{-1}$ curve. We tested 7 devices, taking 17 tests for data analysis.

Figure 14 displays the results of these quantitative experiments. We find the experimental curves fit well with the computational model with a high R-square. We then estimate the proportional coefficients with the curve fitting results, and estimate the decay coefficients with equations based on experiments.

\section{APPLICATIONS}

\section{Embodied Interaction / Learning}

Venous Materials can dynamically visualize people's bodily actions, which can be used for embodied expression, learning, and augmentation. For example, the shoe-sole prototypes demonstrates the utility of embodied learning with body-worn Venous Materials (see Figure 15a, b). This prototype is designed to visualize pressure applied on the shoe sole, while the fluidic display is located on the top of the shoe. It has the advantages being flexible and attachable, which makes it easy for users to attach or embed to their shoes. By visualizing the invisible pressure to users, this can be used to raise awareness of their body balance and motion for rehabilitation and dance lessons. Future challenges include improving visibility by scaling the display area.

As shown in Figure 15c, not only pressure force but also bending motion of the body can be an input. As bending joints of bodies is crucially important for a variety of rehabilitation and training tasks, Venous Materials can be applied in such scenarios as passive responsive materials.

The Venous Materials can also be attached to physical objects and tools to display bodily interaction to them. The two

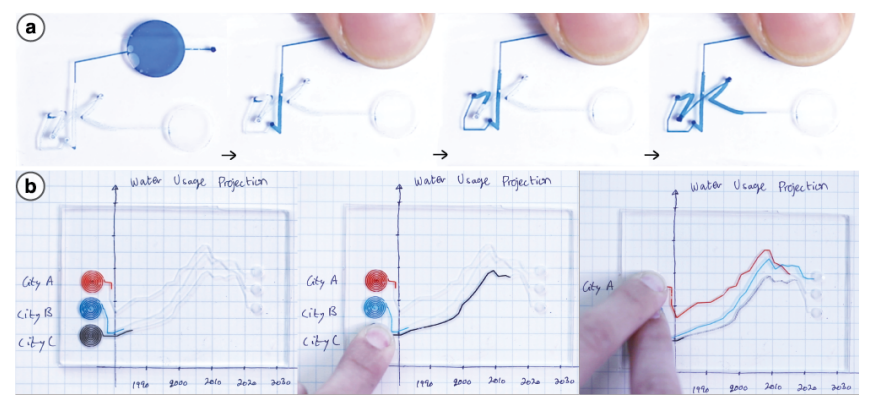

Figure 16. Application of Dynamic Information Representation for (a) Chinese Character Writing, (b) Line Chart.

following applications intend to give colorful expression to artistic motion such as playing the piano and drawing. While both drawing and playing music are artistic fields, learning process often requires an analytic observation on professionals during their performance. For augmenting piano keys (Figure 15d) and a paint brush (Figure 15e), by display of pressure and intensity can visualize additional layer of information to the student that observe the master. Furthermore, in piano, intensity and pressure has linear relations with the playing speed. We envision this tool being used earlier on in the learning process when users slowly press the keys in order to learn the appropriate striking force. Similar to Soter's approach to capture the fluid flow with camera system [63], the change of color on Venous Materials can be captured with a camera to digitally track a user's kinetic action for annotating, and archiving (Figure 15d shows our prototype of such computer vision app).

\section{Object Condition Indicator for Packaging}

There has been an industrial demand in tracking / indicating package status with passive devices [61]. Using the Venous Materials' irreversible property and capability of pressure memory, users could track customized information such as the amount and specific location of force or impact applied to the package. This way, an object's status can be conveyed on the outside of its package so that deliverers or sales store representatives can be aware of the content's condition without opening the packaging (Figure 15f). Real-time representation of internal weight distribution of package could also be useful to ensure the status of fragile products without opening it. 


\section{Dynamic Information Representation}

As the value of dynamic data representation and physicalization is being considered in a recent paper [26], Venous Materials can be used to present dynamic data by encoding information in the material itself. Figure 16a shows how the sequential stroke order for the Chinese character for 'water' can be dynamically visualized. Users can gradually apply force to fluidic repository to control and view the dynamic data of writing the character.

Figure 16b presents an interactive line chart, that are activated and animated with pressure input by the user. Here, the sequential flow is representing time and location that can dynamically change. Users can also choose to display multiple curves at a time and compare the progression of the data and its intersection with other curves. Analog fluidic mechanism utilize the advantages of battery-free, self-contained, flexible, and thin nature of the system that allows it to be embedded into a text book, pop up book or children's book.

\section{LIMITATION AND FUTURE WORK}

Here we discuss the general challenge of the design and approach of Venous Materials, and the potential research space which is led by our initial design and technical exploration in this paper towards interactive fluidic mechanisms.

\section{Robust Fabrication Method and Scalability}

While the fabrication approach introduced in our paper is accessible with off-the-shelf equipment, one limitation is the fragility of the material. Porosity of PDMS is also an important challenge, as liquid tends to evaporate after a long period of usage. We are looking for more robust solutions to enable long-term usage, as well as greater precision, and accuracy for Venous Mechanisms. While our current fabrication pipeline relies on manual tasks (e.g. assembly, cleaning, and injection), there is future work at automation and mass production possibilities. Soft 3D printing is a promising direction for designing complex 3D volumetric fluidic structures without manual assembly, as they are becoming more accessible and precise [12]. Microfluidics' mass production methods such as roll to roll microfluidics [17] is also an exciting direction.

More research needs to be done in order to realize large scale fluidic mechanisms. Future challenges include addressing the accumulating forces of the material on itself and high pressure in the channels. In terms of applications, scaling the display area will include improving visibility of the mechanism and open a space for larger applications including larger wearables (e.g. dance wear, or fashion) and interior products (e.g. carpet or wallpaper).

\section{Design Tool and Simulation}

While our design tool allows relatively accurate simulation of fluidic mechanisms, there is a room for future improvements. Factors such as the surface condition of channels, local deformation of the substrate and the thickness of the device could be taken into consideration for more accurate and precise design. Similarly, connected multi-layer composition and intersects can currently be designed but not simulated. At this time, the design tool can only be used for pressure and bending input.
Other types of deformation inputs can be addressed in future simulation tools and algorithms.

\section{Expanding Interactive Properties and Computation}

The future of fluidic mechanisms holds a rich interaction space for tangible and embodied interfaces with a device composed only of passive materials. As we discussed about the basic architecture and potential design properties of Venous Materials in Figure 2, there are many interaction design opportunities that may be extended with future design properties. Experimenting with different types of Liquid Material properties is a promising direction, as, in $\mathrm{HCI}$, many research has been conducted to utilize material that reacts to heat [56], $\mathrm{pH}$ [30], electric field (using liquid metal / electrorheological fluids) [37, 60, 65, 27], magnetic field (magnetorheological fluids) $[71,81,57,27]$, or UV light [53]. We believe that by combining these special liquid materials with our fluid mechanism design, new interaction opportunities will open up not only for color changing displays, but also for dynamic behaviors in shape, stiffness, temperature and other interactive properties in response to tangible deformation inputs.

In addition to extending interactive properties with special material properties, deeper exploration of the computational logic enabled by fluidic structures is another exciting direction for Venous Materials. Microfluidics research holds an in-depth knowledge of complex integrated logic design [82]. By utilizing such knowledge, it could be extended to incorporate complex logic that would open up the interactivity of the material (e.g. counting the number of deformations, triggering different output patterns in response to the type of deformation inputs [IF THEN states], etc.). We envision a complex network of computational logic to also be embedded in the fluidic structures together with input and output functionalities. We believe that such potential capability of Venous Materials that fully couples I/O and computation enabled with composition of flexible and fluidic material would open up a new design paradigm for the domain of Interactive Matter and User Interfaces.

\section{CONCLUSION}

In conclusion, our paper introduced Venous Materials, a new category of interactive material that utilizes internal fluidic mechanisms that respond to deformation input and displays information by flow of liquid. We presented the design space to define the versatile capability of our approach together with a design pipeline including simulation software and an accessible laser-cutter-based fabrication method. Our technical evaluation validated that the simulation results match the behavior of our actual fabricated samples with simple geometric patterns. While there are many future challenges and design opportunities, the exploration of Venous Materials in this paper opens a channel towards the rich potential interaction capabilities of fluidic mechanisms.

\section{ACKNOWLEDGMENTS}

We would like to thank Libby Rickman for contributing to the experiments setup and data capture; professor Irmgard Bischofberger, professor Zach Lieberman and professor Mathias Kolle for their time and professional advice; Sen Dai for 
advising on design tool development; Daniel Levine, João Wilbert, Jifei Ou, Guni Kofman, Sanad Bushnaq and Shruteja Sharma for advising and brainstorming; Zhipeng Liang, Paula Aguilera and Jonathan Williams in helping with capturing video and images of the prototypes; Deema Qashat, Jack Forman and Joanne Leong for reviewing this paper.

\section{REFERENCES}

[1] Janelle R Anderson, Daniel T Chiu, Rebecca J Jackman, Oksana Cherniavskaya, J Cooper McDonald, Hongkai Wu, Sue H Whitesides, and George M Whitesides. 2000. Fabrication of topologically complex three-dimensional microfluidic systems in PDMS by rapid prototyping. Analytical chemistry 72, 14 (2000), 3158-3164.

[2] Moritz Bächer, Emily Whiting, Bernd Bickel, and Olga Sorkine-Hornung. 2014. Spin-It: Optimizing Moment of Inertia for Spinnable Objects. ACM Trans. Graph. 33, 4, Article Article 96 (July 2014), 10 pages. DOI : http://dx.doi .org/10.1145/2601097.2601157

[3] Christoph Bader, William G Patrick, Dominik Kolb, Stephanie G Hays, Steven Keating, Sunanda Sharma, Daniel Dikovsky, Boris Belocon, James C Weaver, Pamela A Silver, and others. 2016. Grown, printed, and biologically augmented: An additively manufactured microfluidic wearable, functionally templated for synthetic microbes. $3 D$ Printing and Additive Manufacturing 3, 2 (2016), 79-89.

[4] Patrick Baudisch, Stefanie Mueller, and others. 2017. Personal fabrication. Foundations and Trends ${ }^{\circledR}$ in Human-Computer Interaction 10, 3-4 (2017), 165-293.

[5] Alberto Boem and Giovanni Maria Troiano. 2019. Non-Rigid HCI: A Review of Deformable Interfaces and Input. In Proceedings of the 2019 on Designing Interactive Systems Conference. ACM, 885-906.

[6] Jungil Choi, Roozbeh Ghaffari, Lindsay B Baker, and John A Rogers. 2018. Skin-interfaced systems for sweat collection and analytics. Science advances 4, 2 (2018), eaar3921.

[7] Jean-Baptiste Chossat, Hee-Sup Shin, Yong-Lae Park, and Vincent Duchaine. 2015. Soft tactile skin using an embedded ionic liquid and tomographic imaging. Journal of Mechanisms and Robotics 7, 2 (2015), 021008 .

[8] David C Duffy, J Cooper McDonald, Olivier JA Schueller, and George M Whitesides. 1998. Rapid prototyping of microfluidic systems in poly (dimethylsiloxane). Analytical chemistry 70, 23 (1998), 4974-4984.

[9] Don Samitha Elvitigala, Denys JC Matthies, Löic David, Chamod Weerasinghe, and Suranga Nanayakkara. 2019. GymSoles: Improving Squats and Dead-Lifts by Visualizing the User's Center of Pressure. In Proceedings of the 2019 CHI Conference on Human Factors in Computing Systems. ACM, 174.
[10] Sean Follmer, Daniel Leithinger, Alex Olwal, Akimitsu Hogge, and Hiroshi Ishii. 2013. inFORM: dynamic physical affordances and constraints through shape and object actuation.. In Uist, Vol. 13. 417-426.

[11] Jack Forman, Taylor Tabb, Youngwook Do, Meng-Han Yeh, Adrian Galvin, and Lining Yao. 2019. ModiFiber: Two-Way Morphing Soft Thread Actuators for Tangible Interaction. In Proceedings of the 2019 CHI Conference on Human Factors in Computing Systems. ACM, 660.

[12] FormLabs. FormLabs 3D Printer. https://formlabs.com/ - accessed Sep-20-2019.

[13] Hiroshi Fudouzi and Tsutomu Sawada. 2006. Photonic rubber sheets with tunable color by elastic deformation. Langmuir 22, 3 (2006), 1365-1368.

[14] M Garrad, G Soter, AT Conn, H Hauser, and J Rossiter. 2019. A soft matter computer for soft robots. Science Robotics 4, 33 (2019), eaaw6060.

[15] Luc Geurts and Vero Vanden Abeele. 2012. Splash controllers: game controllers involving the uncareful manipulation of water. In Proceedings of the Sixth International Conference on Tangible, Embedded and Embodied Interaction. ACM, 183-186.

[16] Tomoko Hashida, Yasuaki Kakehi, and Takeshi Naemura. 2011. Photochromic sculpture: volumetric color-forming pixels. In ACM SIGGRAPH 2011 Emerging Technologies. ACM, 11.

[17] Jussi Hiltunen, Christina Liedert, Marianne Hiltunen, Olli-Heikki Huttunen, Johanna Hiitola-Keinänen, Sanna Aikio, Mikko Harjanne, Marika Kurkinen, Leena Hakalahti, and Luke P. Lee. 2018. Roll-to-roll fabrication of integrated PDMS-paper microfluidics for nucleic acid amplification. Lab Chip 18 (2018), 1552-1559. Issue 11. DOI : http://dx.doi.org/10.1039/C8LC00269]

[18] Andrew W Holle, Shih-Hui Chao, Mark R Holl, Jeffrey M Houkal, and Deirdre R Meldrum. 2007. Characterization of Program Controlled CO 2 Laser-Cut PDMS Channels for Lab-on-a-chip Applications. In 2007 IEEE International Conference on Automation Science and Engineering. IEEE, 621-627.

[19] Yuki Inoue, Yuichi Itoh, and Takao Onoye. 2018. TuVe: a flexible display with a tube. In SIGGRAPH Asia 2018 Emerging Technologies. ACM, 16.

[20] Alexandra Ion, Johannes Frohnhofen, Ludwig Wall, Robert Kovacs, Mirela Alistar, Jack Lindsay, Pedro Lopes, Hsiang-Ting Chen, and Patrick Baudisch. 2016. Metamaterial mechanisms. In Proceedings of the 29th Annual Symposium on User Interface Software and Technology. ACM, 529-539.

[21] Alexandra Ion, Robert Kovacs, Oliver S Schneider, Pedro Lopes, and Patrick Baudisch. 2018. Metamaterial Textures. In Proceedings of the 2018 CHI Conference on Human Factors in Computing Systems. ACM, 336. 
[22] Alexandra Ion, Ludwig Wall, Robert Kovacs, and Patrick Baudisch. 2017. Digital mechanical metamaterials. In Proceedings of the 2017 CHI Conference on Human Factors in Computing Systems. ACM, 977-988.

[23] Hiroshi Ishii, Dávid Lakatos, Leonardo Bonanni, and Jean-Baptiste Labrune. 2012. Radical atoms: beyond tangible bits, toward transformable materials. interactions 19, 1 (2012), 38-51.

[24] Hiroshi Ishii, Daniel Leithinger, Sean Follmer, Amit Zoran, Philipp Schoessler, and Jared Counts. 2015. Transform: Embodiment of radical atoms at milano design week. In Proceedings of the 33rd Annual ACM Conference Extended Abstracts on Human Factors in Computing Systems. ACM, 687-694.

[25] Hiroshi Ishii and Brygg Ullmer. 1997. Tangible bits: towards seamless interfaces between people, bits and atoms. In Proceedings of the ACM SIGCHI Conference on Human factors in computing systems. ACM, 234-241.

[26] Yvonne Jansen, Pierre Dragicevic, Petra Isenberg, Jason Alexander, Abhijit Karnik, Johan Kildal, Sriram Subramanian, and Kasper Hornbæk. 2015. Opportunities and challenges for data physicalization. In Proceedings of the 33rd Annual ACM Conference on Human Factors in Computing Systems. ACM, 3227-3236.

[27] Yvonne Jansen, Thorsten Karrer, and Jan Borchers. 2010. MudPad: Localized Tactile Feedback on Touch Surfaces. In Adjunct Proceedings of the 23nd Annual ACM Symposium on User Interface Software and Technology (UIST'10). Association for Computing Machinery, New York, NY, USA, 385-386. DOI : http://dx.doi.org/10.1145/1866218.1866232

[28] Wonjin Jeon and Chee Burm Shin. 2009. Design and simulation of passive mixing in microfluidic systems with geometric variations. Chemical engineering journal 152, 2-3 (2009), 575-582.

[29] Yuhua Jin, Isabel Qamar, Michael Wessely, Aradhana Adhikari, Katarina Bulovic, Parinya Punpongsanon, and Stefanie Mueller. 2019. Photo-Chromeleon: Re-Programmable Multi-Color Textures Using Photochromic Dyes. In Proceedings of the 32nd Annual ACM Symposium on User Interface Software and Technology. ACM, 701-712.

[30] Viirj Kan, Emma Vargo, Noa Machover, Hiroshi Ishii, Serena Pan, Weixuan Chen, and Yasuaki Kakehi. 2017. Organic primitives: Synthesis and design of $\mathrm{pH}$-reactive materials using molecular I/O for sensing, actuation, and interaction. In Proceedings of the 2017 CHI Conference on Human Factors in Computing Systems. ACM, 989-1000.

[31] Hsin-Liu Cindy Kao, Manisha Mohan, Chris Schmandt, Joseph A Paradiso, and Katia Vega. 2016. Chromoskin: Towards interactive cosmetics using thermochromic pigments. In Proceedings of the 2016 CHI Conference Extended Abstracts on Human Factors in Computing Systems. ACM, 3703-3706.
[32] Byron Lahey, Audrey Girouard, Winslow Burleson, and Roel Vertegaal. 2011. PaperPhone: understanding the use of bend gestures in mobile devices with flexible electronic paper displays. In Proceedings of the SIGCHI Conference on Human Factors in Computing Systems. ACM, 1303-1312.

[33] Cecilia Laschi. 2017. Octobot-A robot octopus points the way to soft robotics. IEEE Spectrum 54, 3 (2017), 38-43.

[34] David W Lee and Kevin S Gould. 2002. Why leaves turn red: pigments called anthocyanins probably protect leaves from light damage by direct shielding and by scavenging free radicals. American Scientist 90, 6 (2002), 524-531.

[35] Johnny C Lee, Scott E Hudson, and Edward Tse. 2008. Foldable interactive displays. In Proceedings of the 21st annual ACM symposium on User interface software and technology. ACM, 287-290.

[36] Hao-Bing Liu and Hai-Qing Gong. 2009. Templateless prototyping of polydimethylsiloxane microfluidic structures using a pulsed CO2 laser. Journal of Micromechanics and Microengineering 19, 3 (2009), 037002.

[37] Qiuyu Lu, Chengpeng Mao, Liyuan Wang, and Haipeng Mi. 2016. Lime: Liquid metal interfaces for non-rigid interaction. In Proceedings of the 29th Annual Symposium on User Interface Software and Technology. ACM, 449-452.

[38] Qiuyu Lu, Jifei Ou, João Wilbert, André Haben, Haipeng Mi, and Hiroshi Ishii. 2019. milliMorph-Fluid-Driven Thin Film Shape-Change Materials for Interaction Design. In Proceedings of the 32nd Annual ACM Symposium on User Interface Software and Technology. ACM, 663-672.

[39] Stephen A Morin, Robert F Shepherd, Sen Wai Kwok, Adam A Stokes, Alex Nemiroski, and George M Whitesides. 2012. Camouflage and display for soft machines. Science 337, 6096 (2012), 828-832.

[40] Sara Nabil, Jan Kučera, Nikoletta Karastathi, David S Kirk, and Peter Wright. 2019. Seamless Seams: Crafting Techniques for Embedding Fabrics with Interactive Actuation. In Proceedings of the 2019 on Designing Interactive Systems Conference. ACM, 987-999.

[41] Ken Nakagaki, Pasquale Totaro, Jim Peraino, Thariq Shihipar, Chantine Akiyama, Yin Shuang, and Hiroshi Ishii. 2016. Hydromorph: Shape changing water membrane for display and interaction. In Proceedings of the TEI'16: Tenth International Conference on Tangible, Embedded, and Embodied Interaction. ACM, 512-517.

[42] Jifei Ou, Gershon Dublon, Chin-Yi Cheng, Felix Heibeck, Karl Willis, and Hiroshi Ishii. 2016. Cilllia: 3D printed micro-pillar structures for surface texture, actuation and sensing. In Proceedings of the $2016 \mathrm{CHI}$ Conference on Human Factors in Computing Systems. ACM, 5753-5764. 
[43] Jifei Ou, Zhao Ma, Jannik Peters, Sen Dai, Nikolaos Vlavianos, and Hiroshi Ishii. 2018. KinetiX-designing auxetic-inspired deformable material structures. Computers \& Graphics 75 (2018), 72-81.

[44] Jifei Ou, Mélina Skouras, Nikolaos Vlavianos, Felix Heibeck, Chin-Yi Cheng, Jannik Peters, and Hiroshi Ishii. 2016. aeroMorph-heat-sealing inflatable shape-change materials for interaction design. In Proceedings of the 29th Annual Symposium on User Interface Software and Technology. ACM, 121-132.

[45] Jifei Ou, Lining Yao, Daniel Tauber, Jürgen Steimle, Ryuma Niiyama, and Hiroshi Ishii. 2014. jamSheets: thin interfaces with tunable stiffness enabled by layer jamming. In Proceedings of the 8th International Conference on Tangible, Embedded and Embodied Interaction. ACM, 65-72.

[46] Crystal E Owens and A John Hart. 2018. High-precision modular microfluidics by micromilling of interlocking injection-molded blocks. Lab on a Chip 18, 6 (2018), 890-901.

[47] Y. Park, B. Chen, and R. J. Wood. 2012. Design and Fabrication of Soft Artificial Skin Using Embedded Microchannels and Liquid Conductors. IEEE Sensors Journal 12, 8 (Aug 2012), 2711-2718. DOI : http://dx.doi.org/10.1109/JSEN.2012.2200790

[48] Jayson Paulose, Anne S. Meeussen, and Vincenzo Vitelli. 2015. Selective buckling via states of self-stress in topological metamaterials. Proceedings of the National Academy of Sciences 112, 25 (2015), 7639-7644. DOI: http://dx.doi.org/10.1073/pnas. 1502939112

[49] Panagiotis Polygerinos, Nikolaus Correll, Stephen A Morin, Bobak Mosadegh, Cagdas D Onal, Kirstin Petersen, Matteo Cianchetti, Michael T Tolley, and Robert F Shepherd. 2017. Soft robotics: Review of fluid-driven intrinsically soft devices; manufacturing, sensing, control, and applications in human-robot interaction. Advanced Engineering Materials 19, 12 (2017), 1700016.

[50] Julian Pop. Bit Fall. https://vimeo.com/22396196 accessed Sep-20-2019.

[51] Julian Pop. Bit Flow. https://vimeo.com/22390871 accessed Sep-20-2019.

[52] Romain Prévost, Emily Whiting, Sylvain Lefebvre, and Olga Sorkine-Hornung. 2013. Make It Stand: Balancing Shapes for 3D Fabrication. ACM Trans. Graph. 32, 4, Article Article 81 (July 2013), 10 pages. DOI : http://dx.doi.org/10.1145/2461912.2461957

[53] Parinya Punpongsanon, Xin Wen, David S. Kim, and Stefanie Mueller. 2018. ColorMod: Recoloring 3D Printed Objects Using Photochromic Inks. In Proceedings of the 2018 CHI Conference on Human Factors in Computing Systems (CHI'18). ACM, New York, NY, USA, Article 213, 12 pages. DOI : http://dx.doi.org/10.1145/3173574.3173787
[54] Isabel PS Qamar, Rainer Groh, David Holman, and Anne Roudaut. 2018. HCI meets Material Science: A Literature Review of Morphing Materials for the Design of Shape-Changing Interfaces. In Proceedings of the 2018 CHI Conference on Human Factors in Computing Systems. ACM, 374.

[55] Jonathan T Reeder, Jungil Choi, Yeguang Xue, Philipp Gutruf, Justin Hanson, Mark Liu, Tyler Ray, Amay J Bandodkar, Raudel Avila, Wei Xia, and others. 2019. Waterproof, electronics-enabled, epidermal microfluidic devices for sweat collection, biomarker analysis, and thermography in aquatic settings. Science advances 5, 1 (2019), eaau6356.

[56] Daniela K Rosner, Miwa Ikemiya, Diana Kim, and Kristin Koch. 2013. Designing with traces. In Proceedings of the SIGCHI Conference on Human Factors in Computing Systems. ACM, 1649-1658.

[57] Semin Ryu, Jeong-Hoi Koo, Tae-Heon Yang, Dongbum Pyo, Ki-Uk Kyung, and Dong-Soo Kwon. 2016.

Mechanical and psychophysical performance evaluation of a haptic actuator based on magnetorheological fluids. Journal of Intelligent Material Systems and Structures 27, 14 (2016), 1967-1975. DOI : http://dx.doi.org/10.1177/1045389X15620040

[58] Daniel Saakes, Masahiko Inami, Takeo Igarashi, Naoya Koizumi, and Ramesh Raskar. 2012. Shader printer. In ACM SIGGRAPH 2012 Emerging Technologies. ACM, 18.

[59] Yoshimitsu Sagara, Marc Karman, Atsushi Seki, Mehboobali Pannipara, Nobuyuki Tamaoki, and Christoph Weder. 2019. Rotaxane-Based Mechanophores Enable Polymers with Mechanically Switchable White Photoluminescence. ACS Central Science 5, 5 (2019), 874-881. DOI: http://dx.doi.org/10.1021/acscentsci.9b00173

[60] Deepak Ranjan Sahoo, Timothy Neate, Yutaka Tokuda, Jennifer Pearson, Simon Robinson, Sriram Subramanian, and Matt Jones. 2018. Tangible drops: a visio-tactile display using actuated liquid-metal droplets. In Proceedings of the 2018 CHI Conference on Human Factors in Computing Systems. ACM, 177.

[61] SHOCKWATCH. Impact and tilt indicators. https: //www. shockwatch. com. au/product-category/impact-tilt/ - accessed Sep-20-2019.

[62] Ralph C Smith. 2005. Smart material systems: model development. SIAM.

[63] Gabor Soter, Martin Garrad, Andrew T Conn, Helmut Hauser, and Jonathan Rossiter. 2019. Skinflow: A soft robotic skin based on fluidic transmission. In 2019 2nd IEEE International Conference on Soft Robotics (RoboSoft). IEEE, 355-360.

[64] Skylar Tibbits. 2014. 4D printing: multi-material shape change. Architectural Design 84, 1 (2014), 116-121. 
[65] Yutaka Tokuda, Jose Luis Berna Moya, Gianluca Memoli, Timothy Neate, Deepak Ranjan Sahoo, Simon Robinson, Jennifer Pearson, Matt Jones, and Sriram Subramanian. 2017. Programmable liquid matter: 2D shape deformation of highly conductive liquid metals in a dynamic electric field. In Proceedings of the 2017 ACM International Conference on Interactive Surfaces and Spaces. ACM, 142-150.

[66] Bhushan J Toley, Jessica A Wang, Mayuri Gupta, Joshua R Buser, Lisa K Lafleur, Barry R Lutz, Elain Fu, and Paul Yager. 2015. A versatile valving toolkit for automating fluidic operations in paper microfluidic devices. Lab on a Chip 15, 6 (2015), 1432-1444.

[67] Udayan Umapathi, Patrick Shin, Ken Nakagaki, Daniel Leithinger, and Hiroshi Ishii. 2018. Programmable droplets for interaction. In Extended Abstracts of the 2018 CHI Conference on Human Factors in Computing Systems. ACM, VS15.

[68] Nobuyuki Umetani, Athina Panotopoulou, Ryan Schmidt, and Emily Whiting. 2016. Printone: Interactive Resonance Simulation for Free-form Print-wind Instrument Design. ACM SIGGRAPH Asia Computer Graphics 35 (09 2016). DOI : http://dx.doi.org/10.1145/2980179.2980250

[69] Roel Vertegaal and Ivan Poupyrev. 2008. Organic user interfaces. Commun. ACM 51, 6 (2008), 26-30.

[70] Dhaval Vyas, Wim Poelman, Anton Nijholt, and Arnout De Bruijn. 2012. Smart material interfaces: a new form of physical interaction. In CHI'12 Extended Abstracts on Human Factors in Computing Systems. ACM, 1721-1726.

[71] Akira Wakita, Akito Nakano, and Nobuhiro Kobayashi. 2011. Programmable blobs: a rheologic interface for organic shape design. In Proceedings of the fifth international conference on Tangible, embedded, and embodied interaction. ACM, 273-276.

[72] Akira Wakita and Midori Shibutani. 2006. Mosaic textile: wearable ambient display with non-emissive color-changing modules. In Proceedings of the 2006 ACM SIGCHI international conference on Advances in computer entertainment technology. ACM, 48.

[73] Guanyun Wang, Tingyu Cheng, Youngwook Do, Humphrey Yang, Ye Tao, Jianzhe Gu, Byoungkwon An, and Lining Yao. 2018. Printed Paper Actuator: A Low-cost Reversible Actuation and Sensing Method for Shape Changing Interfaces. In Proceedings of the 2018 CHI Conference on Human Factors in Computing Systems. ACM, 569.

[74] Guanyun Wang, Ye Tao, Ozguc Bertug Capunaman, Humphrey Yang, and Lining Yao. 2019. A-line: 4D Printing Morphing Linear Composite Structures. In Proceedings of the 2019 CHI Conference on Human Factors in Computing Systems. ACM, 426.

[75] Guanyun Wang, Lining Yao, Wen Wang, Jifei Ou, Chin-Yi Cheng, and Hiroshi Ishii. 2016. xprint: A modularized liquid printer for smart materials deposition. In Proceedings of the 2016 CHI Conference on Human Factors in Computing Systems. ACM, 5743-5752.

[76] Junchao Wang, Victor G. J. Rodgers, Philip Brisk, and William H. Grover. 2017. Instantaneous simulation of fluids and particles in complex microfluidic devices. PLOS ONE 12 (12 2017), 1-14. DOI :

http://dx.doi.org/10.1371/journal.pone.0189429

[77] L. Wang and E. Whiting. 2016. Buoyancy Optimization for Computational Fabrication. Computer Graphics Forum 35, 2 (2016), 49-58. DOI :

http://dx.doi.org/10.1111/cgf.12810

[78] Wen Wang, Lining Yao, Teng Zhang, Chin-Yi Cheng, Daniel Levine, and Hiroshi Ishii. 2017. Transformative appetite: shape-changing food transforms from $2 \mathrm{D}$ to $3 \mathrm{D}$ by water interaction through cooking. In Proceedings of the 2017 CHI Conference on Human Factors in Computing Systems. ACM, 6123-6132.

[79] Michael Wehner, Ryan L Truby, Daniel J Fitzgerald, Bobak Mosadegh, George M Whitesides, Jennifer A Lewis, and Robert J Wood. 2016. An integrated design and fabrication strategy for entirely soft, autonomous robots. Nature 536, 7617 (2016), 451.

[80] Mark Weiser. 1999. The computer for the 21 st century. ACM SIGMOBILE mobile computing and communications review 3, 3 (1999), 3-11.

[81] Tom White. 1998. Introducing liquid haptics in high bandwidth human computer interfaces. Ph.D. Dissertation. Massachusetts Institute of Technology.

[82] George M Whitesides. 2006. The origins and the future of microfluidics. Nature 442, 7101 (2006), 368.

[83] Mikael Wiberg, Hiroshi Ishii, Paul Dourish, Daniela Rosner, Anna Vallgårda, Petra Sundström, Tobie Kerridge, and Mark Rolston. 2012. Material interactions: from atoms \& bits to entangled practices. In CHI'12 Extended Abstracts on Human Factors in Computing Systems. ACM, 1147-1150.

[84] Lining Yao, Ryuma Niiyama, Jifei Ou, Sean Follmer, Clark Della Silva, and Hiroshi Ishii. 2013. PneUI: pneumatically actuated soft composite materials for shape changing interfaces. In Proceedings of the 26th annual ACM symposium on User interface software and Technology. ACM, 13-22.

[85] Lining Yao, Jifei Ou, Chin-Yi Cheng, Helene Steiner, Wen Wang, Guanyun Wang, and Hiroshi Ishii. 2015. BioLogic: natto cells as nanoactuators for shape changing interfaces. In Proceedings of the 33rd Annual ACM Conference on Human Factors in Computing Systems. ACM, 1-10.

[86] Joo Chuan Yeo, Zhuangjian Liu, Zhi-Qian Zhang, Pan Zhang, Zhiping Wang, and Chwee Teck Lim. 2017. Wearable mechanotransduced tactile sensor for haptic perception. Advanced Materials Technologies 2, 6 (2017), 1700006. 